\title{
Evaluación de la Estrategia de Comunicación "Mejor Hablemos" para promover la Convivencia Pacífica en Cali, 1996-2000
}

Jorge M. Rodriguez ${ }^{1}$, Edgar Muñoz ${ }^{2}$, Andres Fandiño-Losada ${ }^{3}$ y Maria I. Gutiérrez ${ }^{1}$ Médico. M. Sc. Epidemiología. Secretaria de Salud Departamental del Valle del Cauca. E-mail: georod5@hotmail.com georod5@gmail.com

2Estadístico. Universidad del Valle. Cisalva. E-mail: edgarmoz@univalle.edu.co

${ }^{3}$ Médico. M. Sc. Epidemiología. Ministerio de la Protección Social. E-mail: andresfandino@yahoo.com ${ }^{4}$ Médica. Ph. D. Epidemiología. Universidad del Valle. Instituto Cisalva. E-mail: mgutierr@univalle.edu.co

Recibido 7 Marzo 2006/Enviado para Modificación 26 Junio 2006/Aceptado 20 Octubre 2006

\section{RESUMEN}

Objetivos Evaluar la estrategia de comunicación "Mejor Hablemos" para prevenir la violencia y promover la convivencia pacífica en Cali, Colombia. Materiales y Métodos En Cali, entre Agosto de 1996 y Septiembre de 1998, se desarrolló la estrategia a través de dos componentes de intervención: uno poblacional, usando medios masivos de comunicación y otro comunitario, utilizando los medios de comunicación autóctonos de las Comunas 13 y 20. Para determinar el impacto de la estrategia, se hizo una medición post-intervención en el año 2000 y se comparó con la línea de base del estudio ACTIVA, realizada en 1996 por la OPS en ocho ciudades de Latinoamérica (incluida Cali) y España. Se realizaron tres tipos de análisis: descriptivo-comparativo entre los periodos, y análisis bivariado y multivariado usando actitudes y habilidades como variables dependientes.

Resultados Las habilidades mejoraron a nivel poblacional aunque no cambiaron en las comunas intervenidas. En las actitudes hubo cambios favorables en las áreas comunitarias y permanecieron igual a nivel poblacional; los mecanismos para resolver conflictos presentaron una mejora, sugiriendo un aumento de tolerancia de 1996 al 2000, en hombres mayores de la Comuna 20 y resto de Cali.

Discusión "Mejor Hablemos" tuvo poco impacto en las áreas comunitarias intervenidas; sin embargo, la intervención universal generó un impacto positivo sobre el resto de Cali. Para abordar el problema multifactorial de la violencia en los países de Latinoamérica, es necesario poner en marcha procesos de promoción y prevención multisectorial, interdisciplinaria, continua y prolongada, que involucren de forma articulada al individuo y sus contextos familiar, comunitario e institucional-gubernamental. 
Palabras Clave: Prevención, violencia, evaluación, estrategias de intervención, comunicación, actitudes, habilidades (fuente: DeCS, BIREME).

\section{ABSTRACTS \\ Evaluating the "It's better if we talk" (Mejor Hablemos) communication strategy for promoting pacific co-existence in Cali, Colombia, between 1996-2000}

Objective Evaluating the "It's better if we talk" communication strategy for preventing violence and promoting pacific coexistence in Cali, Colombia.

Materials and Methods The strategy was developed through two intervention components in Cali between August 1996 and September 1998: the population (using the mass media) and the community (using Commune 13 and 20's local media). Post-intervention measurement was made in 2000 to determine the strategy's impact and compared to PAHO's 1996 ACTIVA base-line study carried out in eight Latin-American cities (including Cali) and Spain. 3 types of analysis were used: descriptive-comparative between the periods and bivariate and multivariate analysis using attitudes and abilities as dependent variables. Results Abilities improved at population level, although they did not change in the communes taking part. Favourable changes occurred in attitudes in community areas, though remaining equal at population level. The mechanisms for solving conflicts displayed an improvement, suggesting increased tolerance from 1996 to 2000 in older men from Commune 20 and the rest of Cali.

Discussion "It's better if we talk" had little impact on the community areas taking part; nevertheless, the universal intervention had a positive impact on the rest of Cali. Interdisciplinary, ongoing multi-sector promotion and prevention must be embarked on for approaching the multifactor problem of violence in Latin-American countries involving individuals and their family, community and institutional-governmental contexts.

Key Words: Prevention, violence, evaluation, intervention strategy, communication, attitude, ability (source: $M e S H, N L M$ ).

工 a Estrategia de Promoción de la salud "Mejor hablemos" (1), tenía como objetivos generar herramientas para la prevención de la violencia, la promoción de la convivencia, la resolución de conflictos de formapacífica y el desarrollo de habilidades específicas para resolver problemas a través de formas no violentas; cambiar las percepciones subjetivas sobre normas sociales y practicas violentas, teniendo en cuenta que el comportamiento violento esta controlado, al menos en parte, por normas y actitudes sociales. La violencia ha sido el principal problema de salud pública 
que ha tenido Colombia en los últimos 15 años (2); durante la década de los noventa se presentaron tasas de homicidios entre 70 a 80 por 100000 habitantes, las más altas en los departamentos de Antioquia y el Valle del Cauca. En la misma década en América Latina se encontraron tasas promedios de 20 por 100000 habitantes, con Colombia y El Salvador en los primeros lugares (3). La estrategia de comunicación "Mejor Hablemos" se implementó en Cali, debido a que en los primeros años de la década del noventa las tasas de homicidios habían aumentado considerablemente, principalmente a expensas de conflictos de convivencia en las comunas 13 y 20 (4) afectando a los hombres entre los 15 y 24 años. La estrategia se basó con los postulados de la Teoría del Aprendizaje Social de Bandura $(5,6)$ y se centró en los procesos de aprendizaje por observación y refuerzo. Esta teoría sostiene que los seres humanos adquieren destrezas y conductas de modo operante e instrumental y que entre la observación y la imitación intervienen factores cognitivos que ayudan al sujeto a decidir si lo observado se imita o no.

Basándose en esta teoría, se buscaba disminuir en el mediano y largo plazo las actitudes y prácticas violentas que llevaban a que las comunas 13 y 20 tuvieran las tasas de homicidios más altas de la ciudad, promoviendo comportamientos de convivencia y el desarrollo de habilidades específicas para resolver los conflictos interpersonales a través de procesos de observación aprendidos e implementados por sus habitantes (1). La investigación evaluativa parte de una línea de base existente que permitió determinar los cambios en las actitudes, conocimientos y prácticas en torno a la convivencia pacífica. La evaluación implicó comparar los resultados de las encuesta realizadas en dos momentos y analizar los resultados de la intervención implementada.

\section{MÉTODOS}

Diseño. El tipo de diseño fue cuasi-experimental, una evaluación de antes y después con grupo control no aleatorizado. La medición de 1996 se comparó con la medición hecha por la encuesta ACTIVA del año 2 000, con el fin de valorar el concepto y la utilidad del programa de intervención social (7). Mc Alister y Vélez (8) plantearon esta estrategia de intervención para reducir la violencia interpersonal como respuesta a las altas tasas registradas en las Comunas 13 y 20 de la ciudad de Cali en 1994 (4).

Población Objeto. La población estuvo conformada por los habitantes de 15 a 70 años de la ciudad de Cali en los años 1996 y 2000. 
Muestra. El tamaño de la muestra (9) se eligió asumiendo un nivel de confianza de 95 \% y un error máximo de 2,8 \%. La muestra de 1996 fue de 1200 personas considerando un $15 \%$ de no respuesta. En Cali se utilizaron técnicas de recolección de información diferentes a las restantes ciudades (10), por lo que la muestra fue de cerca de 3000 personas (50\% de las comunas 13 y 20 y el otro $50 \%$ del resto de la Ciudad). Para el año 2000, la muestra fue de 2610 personas, 46 \% para las comunas 13 y 20 y $54 \%$ para el resto de la ciudad.

Variables. Se utilizaron las siguientes variables: Sociodemográficas. Se registraron la comuna de residencia, edad, sexo, tiempo de residencia en la ciudad (en años), religión y frecuencia de asistencia a la iglesia del entrevistado. Habilidades. Definidas como la capacidad que tenían las personas para manejar una situación: aptitud de la persona ante problemáticas en el hogar (con la pareja, los hijos u otros menores) o en su comunidad. Actitudes. Definidas como las creencias evaluativas ante situaciones asociadas a hechos violentos (comportamientos sociales, infidelidad, abuso sexual, situaciones de tolerancia vs. intolerancia, agresiones a terceros, ajuste de cuentas por cuenta propia, entre otros) eran indicadores indirectos de las conductas de las personas. Resolución de conflictos. Evaluaba la forma como las personas resolvían un conflicto, por medio de métodos legales o con la mediación de un 'tercero' planteando situaciones imaginarias.

Técnicas de recolección de la información. En 1996, las personas se seleccionaron mediante un muestreo multietápico de cinco fases (9). Para el 2000, se siguió el mismo esquema de muestreo que se había adoptado en 1996, tomando las mismas direcciones de los encuestados.

Análisis de datos. Se creó una base de datos utilizando Epi-info 6,04 (11) y SPSS 11.0 (12). La edad y el sexo se manejaron en cuatro grupos siguiendo el enfoque de la epidemiología de la violencia (9): hombres y mujeres, mayores y menores de 35 años. Se determinaron diferencias estadísticamente significativas entre los períodos estudiados empleando pruebas t de Student y Chi cuadrado (13). Los puntajes de las escalas Likert (14) se homogenizaron en las dimensiones de habilidades y actitudes; las respuestas con puntajes altos indicaban tranquilidad o sosiego y con puntajes bajos, agresión o violencia.

Se hizo un análisis comparativo recodificando las variables de las dimensiones de habilidades y actitudes. El análisis multivariado se realizó mediante regresión logística no condicional en cada dimensión de los mecanismos de resolución de 
conflictos (usa/no usa métodos legales); en las dimensiones de habilidades y actitudes se utilizó análisis de covarianza para eliminar las diferencias que alterarían los resultados del diseño, ya que algunas variables se podían comportar como confusoras. Para garantizar el anonimato y la confidencialidad de los entrevistados, el equipo de investigadores de Cisalva creó un formato de Consentimiento Informado (15).

\section{RESULTADOS}

Respecto a las características sociodemográficas se obtuvieron los siguientes resultados:

- Edad. No se encontraron diferencias estadísticamente significativas con los promedios de las edades; en 1996 el promedio fue de 35,8 años y en el 2000 fue de 35,4 (p>0,05).

- Sexo. En 1996 hubo una mayor proporción de población de sexo masculino entrevistado; las diferencias entre hombres y mujeres no fueron significativas.

- Comunas agrupadas. Cali se compone de 20 comunas urbanas; para el 2000 hubo mas entrevistados del resto de Comunas de la ciudad (53 $\%)$ presentando una diferencia estadísticamente significante entre las dos mediciones.

- Religión. Para 1996 según la religión profesada, se encontró que, 2 455 (84,5 \%) eran católicos, y en el 2000 se encontró que esta religión tuvo una menor representación, 1979 (73,9 \%).

- $\quad$ Frecuencia de asistencia a actos litúrgicos. Para 1996 el 36,0 \% dijo que asistía una vez al mes mientras que en el 2000 se evidenció que era mayor.

- Años de vivir en la ciudad. No hubo diferencias estadísticamente significativas, En el 2000 no se indagó por etnia, motivo por el cual no se pudo comparar en los dos períodos.

Habilidades y Actitudes: En la Tabla 2 se encuentran los resultados para 1996 y el 2000. 
Tabla 1. Indicadores sociodemográficos de la investigación del fenómeno de la violencia en Colombia. Santiago de Cali, 1996-2000

\begin{tabular}{clcc}
\hline \multirow{2}{*}{ Variables } & & \multicolumn{1}{c}{ Descripción } & \multicolumn{2}{c}{ Años } \\
\cline { 3 - 4 } & & 1996 & 2000 \\
\hline Sexo del & Masculino & 3,4 & 58,7 \\
Entrevistado & Femenino & 36,6 & 41,3 \\
\hline Comunas por & Comunas 13 y 20 & 51,1 & 46,2 \\
Grupos & Resto de comunas & 48,9 & 53,8 \\
\hline \multirow{2}{*}{ Grupos de edad } & Jóvenes & 53,8 & 51,4 \\
& Mayores & 46,2 & 48,6 \\
\hline \multirow{4}{*}{ Religión que profesa Otra religión } & 5,8 & 10,4 \\
& Protestante o evangélica & 84,5 & 73,9 \\
& No tiene religión & 2,9 & 7,4 \\
& No cree en Dios & 6,4 & 7,9 \\
& Mas de una vez por semana & 0,4 & 0,4 \\
\hline \multirow{2}{*}{ Frecuencia con que } & Al menos una vez por semana & 17,8 & 15,6 \\
asiste a la iglesia & Menos de una vez por mes & 13,3 & 15,6 \\
& Nunca asiste & 36,0 & 27,8 \\
& Valores perdidos & 19,6 & 17,5 \\
& 0 a 4 años & 7,2 & 3,0 \\
\hline \multirow{2}{*}{ Años de vivir en } & 5 a 10 años & 6,9 & 1,8 \\
la ciudad & 11 ó más años & 8,0 & 7,9 \\
& Valores Perdidos & 9,6 & 0,3 \\
\hline
\end{tabular}

Tabla 2. Investigación del fenómeno de la violencia en Colombia: Habilidades y actitudes en Santiago de Cali, 1996.

\begin{tabular}{|c|c|c|c|c|c|}
\hline Item & Variable & & 1996 & & 000 \\
\hline \multirow{7}{*}{ 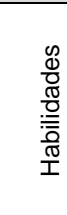 } & & No. & Promedio & No. & Promedio \\
\hline & Autocontrol para no pelear & 2904 & 4,14 & 2607 & 4,18 \\
\hline & Perder el Control ante un desacuerdo & 2905 & 3,35 & 2604 & 3,27 \\
\hline & Forma de afrontar una agresión ajena & 2905 & 3,46 & 2588 & 3,36 \\
\hline & Formas de afrontar un problema con la pareja & 2892 & 4,09 & 2597 & 4,13 \\
\hline & Modo de corregir a un menor & 2892 & 4,14 & 2604 & 4,26 \\
\hline & Método para corregir y explicar a un menor & 2899 & 4,40 & 2609 & 4,55 \\
\hline \multirow{9}{*}{ 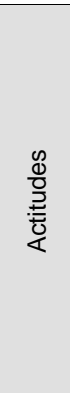 } & Posición cuando alguien no respeta una fila & 2902 & 2,46 & 2610 & 2,56 \\
\hline & $\begin{array}{l}\text { Actitud cuando hieren a alguna persona por } \\
\text { infidelidad }\end{array}$ & 2986 & 2,99 & 2605 & 3,01 \\
\hline & $\begin{array}{l}\text { Actitud ante la muerte a alguien que ha seducido } \\
\text { a familiar }\end{array}$ & 2987 & 3,18 & 2593 & 3,18 \\
\hline & $\begin{array}{l}\text { Posición cuando se arreglan "cuentas de dinero" } \\
\text { con armas y sicarios }\end{array}$ & 2898 & 3,49 & 2605 & 3,46 \\
\hline & Postura por matar a alguien que ha violado la hija & 2890 & 2,08 & 2604 & 238 \\
\hline & Actitud ante situaciones de "limpieza social" & 2900 & 3,09 & 2601 & 2,85 \\
\hline & Derecho a matar por defender a la familia & 2902 & 3,02 & 2606 & 3,33 \\
\hline & Matar por defender casa o propiedad & 2903 & 3,46 & 2603 & 3,70 \\
\hline & $\begin{array}{l}\text { Actitud ante la muerte de alguien que inició una } \\
\text { pelea }\end{array}$ & 2985 & 3,05 & 2606 & 3,79 \\
\hline
\end{tabular}


Análisis bivariado

Este análisis se llevo acabo con las variables: habilidades y actitudes entre 1996 y 2000, en cada una de los grupos de comunas (intervenidas y no intervenidas). Para la Comuna 13, entre 1996 y 2000 se encontró que en la variable habilidades no hubo diferencias estadísticamente significativas $(p>0,05)$, con puntajes similares entre los periodos; en cambio en la variable actitudes, hubo cambios estadísticamente significativos $(\mathrm{p}<0,001)$, con puntajes mayores en el 2000 respecto a 1996. En la comuna 20 hubo cambios con disminución en los puntajes de 1996 al 2000.

Tabla 3. Habilidades y Actitudes en las Comunas 13 y 20 entre 1996 y 2000

\begin{tabular}{ccccccc}
\hline Variables I & \multicolumn{3}{c}{ Comuna 13 } & \multicolumn{3}{c}{ Comuna 20 } \\
\cline { 2 - 7 } Año & 1996 & 2000 & $\mathrm{P}$ & 1996 & 2000 & $\mathrm{p}$ \\
\hline Habilidades & 4,68 & 4,66 & 0,67 & 4,74 & 4,59 & 0,006 \\
Actitudes & 3,30 & 3,45 & 0,001 & 3,73 & 3,54 & 0,001 \\
\hline
\end{tabular}

Estos resultados fueron concordantes con otros análisis bivariados realizados, donde los cambios en las conductas interpersonales de los habitantes de la comuna 20 entre 1996 y el 2000 fueron escasos o si los hubo fue con descenso en el periodo evaluado. Para el resto de Cali, no hubo diferencias significativas en la variable habilidades; para la variable actitudes, hubo cambios estadísticamente significativos ( $<<0.01)$, con puntajes mayores en el 2000 respecto a 1996.

\section{Análisis multivariado}

Para la dimensión Habilidades, el modelo general fue estadísticamente significativo ( $\mathrm{F}: 13,8 ; \mathrm{gl}=9, \mathrm{p}<0,001)$, aunque solo explicaba un $2,4 \%$ de la variabilidad de las habilidades (R cuadrado 0,024). Las variables independientes corresponden a Intervención y Año. Las covariables son frecuencia con que una persona asiste a la Iglesia, sexo, grupos de edad agregados de 1 a 3 quinquenios, si era nacido o no en Cali, años de vivir en la ciudad, agrupada por 1 ó 2 quinquenios, y las tasas de homicidio para cada comuna en 1996. Al realizarse el Ancova, ajustando por cada una de las posibles variables confusoras o covariables para sexo, grupos de edad, si vivía en la ciudad y tasa de homicidios no hubo diferencias estadísticamente significativas, $\mathrm{p}>0,05$ (sugestivo de no ser factores confusores); las covariables de asistencia a la iglesia y haber nacido en Cali tuvieron diferencias estadísticamente significativas, sugiriendo que se pudieron comportar como confusores en la dimensión de habilidades. 
Figura 1. Ancova para Habilidades entre comunas intervenidas y no intervenidas por Mejor Hablemos, Santiago de Cali, 1996 y 2000

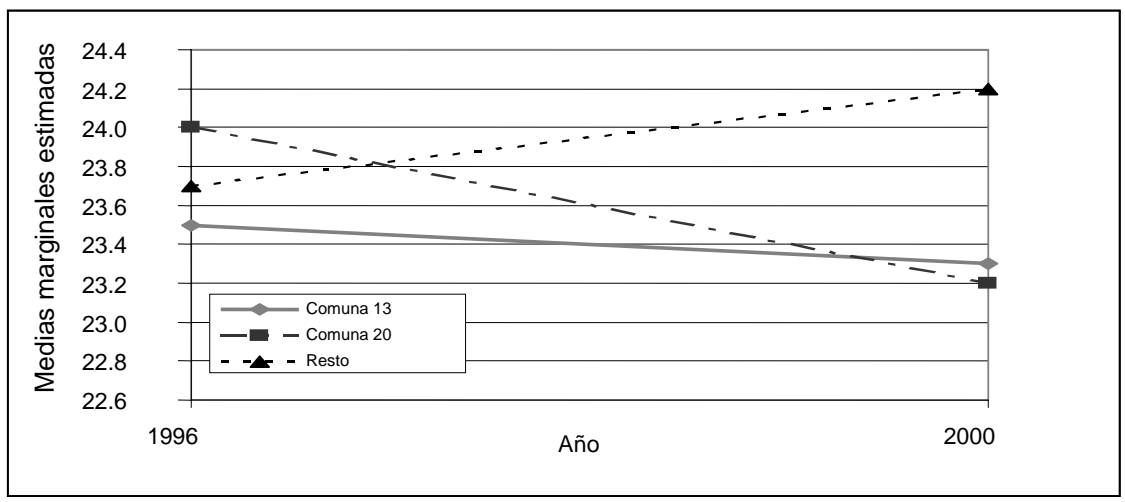

En esta figura se nota el comportamiento de los resultados para la dimensión Habilidades; la Comuna 20 muestra un mayor descenso que la Comuna 13; las comunas resto de Cali evidencian un ascenso notorio, estos resultados son concordantes con el análisis bivariado descrito anteriormente. También se observa, un probable cruce de la variable independiente, antes de 1996, indicando un efecto de interacción entre intervención y año, sugestivo de un cambio del efecto de la intervención para habilidades a través del tiempo.

Para actitudes el modelo general fue estadísticamente significativo (F: 46,2; gl 9; $\mathrm{p}<0,001)$. Al ajustar las variables independientes: La intervención no tuvo diferencia estadísticamente significativas $(\mathrm{p}>0.05)$. Las covariables grupo de edad 15, permanencia y nativo, no presentaron diferencias estadísticamente significativas, $\mathrm{p}>0,05$, (factores no confusores); iglesia, sexo y tasa de homicidios en 1996, tuvieron diferencias estadísticamente significativas, sugiriendo factores de confusión en esta dimensión.

En esta figura se evidencian los resultados de la dimensión actitudes: la comuna 20 muestra un ascenso levemente menor que la comuna 13; las comunas del resto de Cali, no presentan cambio entre los dos periodos. Al igual que con habilidades, estos resultados son similares a los encontrados con el análisis bivariado previamente desarrollado. También en esta dimensión se evidencia un efecto de interacción entre la intervención y la variable año; en la gráfica se observa el cruce de las líneas de la variable independiente entre el periodo de 1996-2000, donde el efecto de la intervención para las actitudes cambia a través del tiempo. 
Figura 2. Ancova para Actitudes entre comunas intervenidas y no Intervenidas por Mejor Hablemos, Santiago de Cali, 1996 y 2000

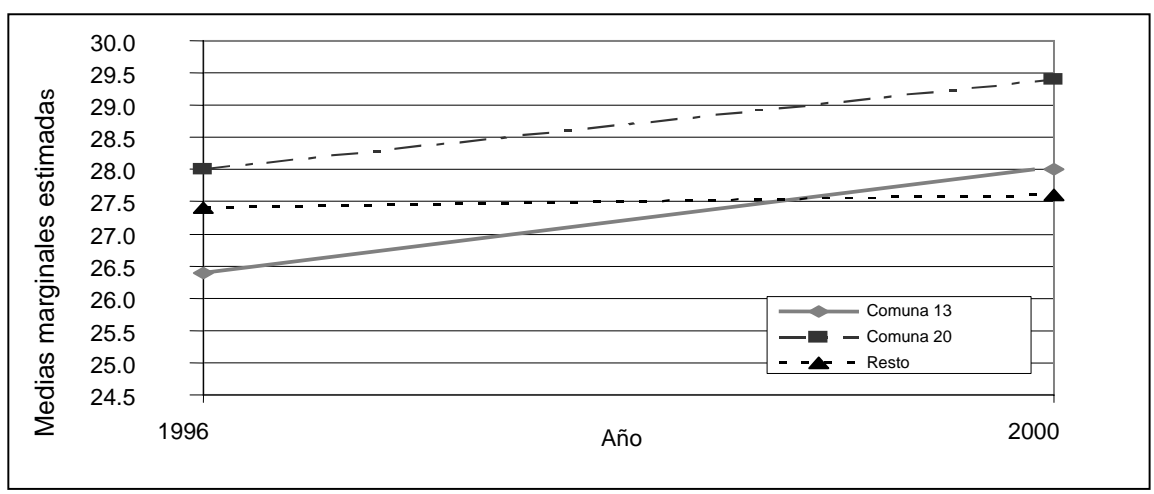

Respecto al resultado de la regresión logística $(13,16)$, para la variable mecanismos de resolución de conflictos que evaluaba el pertenecer a la comuna 13, la probabilidad de demandar fue de 1,2 veces respecto a vivir por fuera de la misma (resto de Cali o comuna 20), ajustada por todas las demás variables que aplicaron para el modelo, siendo estadísticamente significativo. Por pertenecer a la comuna 20 la probabilidad de demandar fue 1,3 veces, siendo también estadísticamente significativo; en el joven la probabilidad de demandar fue 1,2 veces respecto al mayor y en el hombre la probabilidad de demandar fue 1,7 veces respecto a la mujer.

Tabla 4. ODDS RatioRegresión logística en los "Mecanismos de Resolución de conflictos"

\begin{tabular}{|c|c|c|c|c|}
\hline Variables & $\begin{array}{c}\text { Comuna } \\
13\end{array}$ & $\begin{array}{l}\text { Comuna } \\
20\end{array}$ & Edad & Sexo \\
\hline Demanda ante no pago por un trabajo grande & 1,14 & 1,27 & 1,17 & 1,6 \\
\hline $\begin{array}{l}\text { Demanda a alguien que ha lastimado a si mismo o a } \\
\text { un familiar para que pague los daños causados }\end{array}$ & 0,98 & 1,35 & 1,05 & 1,27 \\
\hline $\begin{array}{l}\text { Denuncia ante comisaría a alguien que ha seducido } \\
\text { a familiar menor de } 15 \text { años }\end{array}$ & 0,97 & 1,29 & 0,52 & 0,84 \\
\hline Denuncia a conocido que ha robado objeto de valor & 0,96 & 1,13 & 0,71 & 1,14 \\
\hline Contacto con mediador ante un conflicto por linderos & 1,28 & 1,05 & $0 ., 89$ & 1,08 \\
\hline
\end{tabular}

Respecto a la difusión y reconocimiento de "Mejor Hablemos" en las Comunas con y sin Intervención Comunitaria se pudo determinar las diferencias en el nivel de conocimiento de la Estrategia de Comunicación "Mejor Hablemos" entre las Comunas Intervenidas y no Intervenidas a nivel comunitario. Respondieron a esta variable 2606 personas equivalentes a un 87,6 \% de los encuestados inicialmente programados: 2975. 
Tabla 5. Reconocimiento del logo por Comunas según nivel de intervención

\begin{tabular}{cccc}
\hline \multirow{2}{*}{ Comunas } & \multicolumn{2}{c}{ RECOLOGO $^{1}$} & \\
\cline { 2 - 3 } & $\mathrm{Si}$ & No & Total \\
\hline Intervenida & 387 & 813 & 1200 \\
No intervenida & 264 & 1142 & 1406 \\
Total & 651 & 1955 & 2606 \\
\hline
\end{tabular}

Chi cuadrado $=62,7 \mathrm{p}<0.001 .1$ : RECOLOGO: Corresponde a la variable: Recordaba el Logo empleado como símbolo de Mejor Hablemos

Existió una diferencia estadísticamente significativa $(\mathrm{p}<0.01)$ entre las comunas con y sin intervención comunitaria respecto al reconocimiento del Logo que identificó la Estrategia de Comunicación Comunitaria, con un porcentaje mayor de personas que recordaban el Logo y que vivían en las comunas intervenidas. Hubo diferencias estadísticamente significativa con una $\mathrm{p}<0,01$ entre las Comunas con y sin Intervención comunitaria respecto al conocimiento del Boletín comunitario "Convivencias" usado en la estrategia; dado por un porcentaje mayor de personas que conocían el boletín y que vivían en las comunas con intervención comunitaria.

Tabla 6. Conocimiento del boletín Convivencias por comunas

\begin{tabular}{|c|c|c|c|}
\hline \multirow[t]{2}{*}{ Comuna } & \multicolumn{2}{|c|}{ CONVIVEN $^{1}$} & \multirow[t]{2}{*}{ Total } \\
\hline & Si & No & \\
\hline Intervenida & 318 & 882 & 1200 \\
\hline No intervenida & 228 & 1174 & 1402 \\
\hline Total & 546 & 2056 & 2602 \\
\hline
\end{tabular}

Finalmente en cuanto al recuerdo de historias sobre resolución pacífica de conflictos y pertenecer al grupo de comunas intervenidas o no, hubo una diferencia estadísticamente significativa, $(\mathrm{p}<0,05)$. El porcentaje de personas que recordaron historias, fue mayor en las Comunas intervenidas respecto a las no intervenidas.

\section{DISCUSIÓN}

La violencia, que ha sido el principal problema de salud pública para Colombia en los últimos años $(2,3,17)$, se presenta como un fenómeno sociocultural de características epidémicas, generada por diversas circunstancias o factores de riesgo, manifiesta como un problema complejo y multifacético; algunos autores no hablan de la violencia sino de las violencias debido a los diferentes orígenes y manifestaciones de este fenómeno (18). Investigadores del Ministerio de 
Salud (17) consideran que en Colombia existe un problema sociocultural de intolerancia, manifiesto por "la ley del más fuerte", con la no aceptación de ideas y posiciones de otros.

Franco (19) y otros investigadores $(4,20)$ exponen que en Colombia existen varios elementos estructurales y coyunturales que explican la vigencia y permanencia del problema de violencia a lo largo de la segunda parte del siglo pasado y que han recrudecido el fenómeno en los últimos 20 años en nuestro país. Dentro de los elementos estructurales están la inequidad, la impunidad y la intolerancia. La inequidad manifiesta por el aumento de la relación entre el número de homicidios y el inverso de las Necesidades Básicas Insatisfechas en forma directa; la impunidad reflejada en el aumento de las tasas de delitos, homicidios y la disminución de los detenidos por esos homicidios; la intolerancia reflejada por el aumento de las muertes en combate, contra las personas marginales, los homicidios políticos, las ejecuciones extrajudiciales, al igual que el establecimiento de las masacres. Como elementos coyunturales se exponen el modelo económico neoliberal, la agudización del conflicto político-militar interno y la emergencia y expansión del narcotráfico, este último con actores diversos como guerrilla, paramilitares y fuerzas armadas, generadores de manifestaciones variadas de violencia. Por tanto, estas situaciones han sido generadoras perennes de un fenómeno social complejo de difícil control.

Las propuestas de intervención se han centrado en medidas legislativas y represivas cuya escasa efectividad, en parte por su no-cumplimiento, se refleja en las estadísticas de morbimortalidad por causa externa. En los últimos años se han sumado propuestas educativas que en general no han sido evaluadas. Ante estas circunstancias complejas, investigadores nacionales y regionales $(4,20,21)$ han propuesto abordajes multidisciplinarios e integrales, que tengan en cuenta diferentes elementos para disminuir y enfrentar la inseguridad y la violencia. La estrategia, objeto de esta investigación, pretendió motivar y consolidar procesos de convivencia pacífica, generar entendimiento, compresión y empatía entre los miembros de la sociedad.

"Mejor Hablemos" fue consonante y precursora de las políticas nacionales de convivencia pacífica creadas por el gobierno nacional, se basó en elementos conceptuales de la Teoría del Aprendizaje Social de Bandura (5,6); no obstante, en su implementación y desarrollo se notó indirectamente una mezcla de técnicas en torno a la organización comunitaria, Psicología Social y modelos de cambio de comportamiento-comunicación (22). No obstante sus diseñadores 
enfatizan que el componente conceptual desarrollado se basó exclusivamente en la Teoría del Aprendizaje Social. Las variables sociodemográficos de los encuestados entre los dos períodos en las áreas intervenidas y no intervenidas fueron similares ( $>00,05)$, excepto en el género, religión católica y para el procedente de fuera de Cali, donde la distribución en estos grupos varió entre los dos períodos $(\mathrm{p}<0,001)$. La etnia desafortunamente no fue evaluada para el 2000, impidiendo estratificar o explicar los hallazgos por este factor, reconociendo que en las zonas intervenidas vive más proporción de población Afro $(23,24)$.

La estrategia de comunicación tuvo mayor penetración y fue mejor conocida en las áreas con intervención comunitaria y personalizada que en las áreas que usaron medios masivos de comunicación, debido a que los resultados de las variables que identificaron el Logo, las historias y el boletín Convivencias empleados en "Mejor Hablemos", presentaron diferencias estadísticamente significativas en las áreas intervenidas $(p<0,05)$ respecto a las no intervenidas. La asociación más fuerte se presentó en las Comunas Intervenidas con el Recuerdo del Logo y el Boletín Comunitario Convivencias que con el Recuerdo de Historias, esos se distribuyeron en diferentes sitios: Centros de salud, tiendas, peluquerías, carnicerías, hogares de bienestar familiar, entre otros.

Los resultados de los análisis bivariados y multivariados de "Mejor Hablemos" fueron concordantes en el periodo evaluado (1996 al 2000). Las habilidades: en las áreas intervenidas con medios comunitarios y por procesos interpersonales, (comunas 13 y 20) presentaron un descenso en el puntaje; el descenso fue mayor en la comunas 20 que en la comuna 13; en las áreas sin este tipo de intervención (resto de Cali) hubo un ascenso, sugestivo de mejoría para la resolución pacífica de conflictos. Las actitudes: en las áreas con y sin intervención comunitaria mejoraron, no obstante la mejoría es mayor en las zonas intervenidas (Comunas 13 y 20), principalmente en la comuna 13. Estas dimensiones se evaluaron con Análisis de Covarianza o Ancova, ajustando por sexo, edad agrupada, frecuencia de asistencia a actos religiosos, número de años de vivir en la ciudad y por las tasas de homicidio de 1996, según lugar de residencia. Para habilidades se encontró que nativo e iglesia se comportaron como factores de confusión $(\mathrm{p}<0,001)$ siendo estadísticamente significativos; para actitudes las variables iglesia, sexo y tasas de homicidio de 1996 al ajustarse se comportaron como factores confusores $(p<0,001)$ siendo estadísticamente significativo. Las demás variables no se comportaron como factores de confusión. 
En cuanto a la dimensión mecanismos de resolución de conflictos, se presentó una tendencia a resolver los conflictos por vías legales de 1996 al 2000, principalmente en la comuna 20 y en el resto de Cali, por personas del sexo masculino y mayores de 35 años, esto se puede explicar en parte por la mayor consolidación de redes comunitarias existentes en la comuna 20. La "mejoría" obtenida en las habilidades de todos los grupos de edad - al igual que en el grupo de mujeres mayores de 35 años- del resto de Cali, se pueden atribuir, principalmente, a efectos de maduración. Investigadores de socioeconomía de la Universidad del Valle (21) consideran que factores sociales, económicos y culturales pudieron afectar diferentemente a sectores de la Ciudad. Los habitantes de las comunas no intervenidas (resto de la ciudad) pudieron haberse expuesto durante cuatro años a "mejor" educación, empleos sólidos, mayor seguridad social, mejor consolidación de las estructuras y bases familiares; posiblemente en las Comunas 13 y 20, hubo mayor exposición a hechos generadores de violencia y agresividad, lesiones interpersonales, agresiones verbales, según las normas sociales, familiares, culturales que manejan sus habitantes(18).

Hay dos situaciones de contexto que pueden explicar estos resultados:

1. La participación y capacitación en "Mejor Hablemos" fue dada principalmente, a mujeres, cabezas de hogar, madres comunitarias, interesadas en aprender, hablar y difundir situaciones sobre los temas de convivencia; aunque se crearon diversas estrategias para intentar interesar a diferentes grupos poblacionales, sobre convivencia pacífica, como jóvenes, personas de la tercera edad y algunos estudiantes de colegio. No obstante, los problemas de violencia se dan principalmente entre integrantes del género masculino $(4,25)$.

2. Existen elementos socioculturales que generan una 'cultura de violencia' en las comunas intervenidas: 13 y 20. Se viven situaciones que expresan los límites de la intolerancia e impunidad, la no aceptación de la diferencia, la dificultad de "vivir sin problemas"; se han creado "normas o reglas de convivencia" (18) o más bien de supervivencia, como el no mirar fijamente a los ojos del otro, agachar la cabeza, no entrar en disputas, ni andar por la calle luego de las ocho de la noche, no portar dinero o joyas en la calle; las mujeres, no usar ropa ajustada o minifaldas, aceptar insultos de un joven, no pelear con un vicioso, darle la razón casi todas las veces al otro y en fin, aceptar una serie de imperativos, códigos, lenguajes y expresiones del sector. Dentro de este contexto, las políticas públicas, la acciones de promoción de la convivencia pacífica y prevención de las violencias pueden quedar como "oasis en un desierto"; la posibilidad de 
impactos poblacionales es baja, más aun si no son continuas e articuladas a un enfoque interdisciplinario.

Rubio (26) plantea que los procesos de promoción de convivencia pacífica son de difícil arraigo cuando problemas estructurales afectan a una comunidad, no obstante la solución de fortalecimiento del aparato de justicia del Estado, que él enfatiza, para controlar la violencia, es limitada al no abordar el problema desde una perspectiva global e integral, que involucre elementos intersectoriales; además de fortalecer al aparato de justicia-militar, se necesita promover el desarrollo personal, social comunitario, estimular el derecho a la vida, rechazar la violencia en todas sus manifestaciones como generadora de destrucción e impacto negativo, con procesos continuos, permanentes y a largo plazo (27).

McAlister $(8,28)$ en su revisión de la violencia juvenil en las Américas habla de diferentes procesos exitosos que involucraban programas basados en las escuelas: programas para padres, y pares, con diferentes técnicas; un ejemplo fue el denominado "forjadores de paz", donde hubo una reducción en las lesiones externas y traumas provocados en las escuelas que fueron objeto de intervención respecto a escuelas control. Resalta programas escolares con la mediación de pares, en la cual resocializan a los jóvenes problemas con otros que "tienen buenas costumbres", promocionándoles hábitos saludables y alejándolos de las circunstancias de riesgo como alcoholismo, actos delictivos y estimulándolos en logros académicos.

Ejemplos exitosos como estos permiten sintetizar algunas hipótesis explicativas, propias y externas a la estrategia, que evidencian las dificultades de su impacto social.

\section{A. Internas:}

1. La teoría y el modelo conceptual aplicado pudo ser insuficiente para explicar una sociedad compleja, cargada de factores generadores de violencia.

2. La evaluación de impacto ocurre dos años después la terminación de los recursos, debiéndose haber realizado en un tiempo más cercano a su culminación.

\section{B. Externas:}

1. La estrategia originalmente estaba concebida para ser ejecutada en cinco años (de 1996 a 2001), los recursos los suspende el Ministerio de Salud por dos años, por la crisis institucional de la Universidad del Valle en 1998.

2. En las áreas con intervención comunitaria se han presentado circunstancias 
históricas que han llevado a procesos de exclusión social, inequidad, pobreza e impunidad.

3. La estrategia de comunicación estuvo articulada a políticas públicas intersectoriales de forma parcial, reconociendo que es difícil separar el impacto en un programa de comunicación, de los efectos de otros factores -variables productoras de confusión- sobre las conductas personales (29).

No obstante estas circunstancias, las evidencias de promoción de la salud y prevención de la enfermedad $(30,31)$ han demostrado ser más costo-efectivas en los niveles primarios que en los niveles secundarios y/o terciarios, por tanto estrategias como "Mejor Hablemos" es necesario promoverlas, estimularlas, desarrollarlas y difundirlas dentro de un proceso de "construcción social" acompañadas integralmente con elementos de la política de prevención de la violencia (26) y promoción de la convivencia pacífica para América Latina (32). Es importante preguntarse cuál sería el estado de las comunas y de sus habitantes si estrategias como "Mejor Hablemos" no hubieran motivado a hablar de convivencia pacífica, entendimiento, arreglos sin violencia o por medios pacíficos"

Agradecimientos. Al Ministerio de Salud de Colombia. Instituto CISALVA, Proyecto Propuesta de Promoción de Creencias, Actitudes y Habilidades de Convivencia Pacífica en 15 Comunas de Santiago de Cali.

\section{REFERENCIAS}

1. Muñoz E, Gutiérrez MI, Guerrero R. Evaluación del impacto de una estrategia de Información, Educación y Comunicación para la prevención de la violencia en Bogotá, Cali y Medellín 2003-2004, Ministerio de la Protección SocialUniversidad del Valle, Instituto Cisalva; 2004.

2. Franco. S, El Quinto no matar: Contextos explicativos de la Violencia en Colombia. Santa fe de Bogotá: Tercer Mundo Editores; 2000.

3. Organización Panamericana de la Salud. Análisis de la situación de Salud en las Américas 2000. Washington, D.C.1999; 4:1-5.

4. Espitia VE. Vigilancia epidemiológica de los homicidios ocurridos en Cali durante 1993. La epidemiología aplicada a la administración pública En: Concha A, Carrión F, Cobo G. Ciudad y violencias en América Latina. Quito: Edición conjunta con la alcaldía de Cali y el programa gestión Urbana de las Naciones Unidas; 1994. pp. 154-166.

5. Bandura A. Aggression: A social learning analysis. Englewood Cliffs, N.J: Prentice Hall; 1973.

6. Bandura A. Teoría del Aprendizaje Social. Madrid: Espasa Universitaria; 1982. 
7. Pabón. H. Evaluación en Servicios de Salud. Fundamentos de la Evaluación, Marco Conceptual de la Evaluación Capítulos 1 y 3, Modelo Prides, Universidad del Valle, Facultad de Salud, Cali, 1985. pp. 11-15.

8. McAlister A, Vélez, LF. Conceptos de las ciencias del comportamiento en investigación sobre la prevención de la violencia, Rev Panam Salud Pública, 1999; 5(4/5): 316- 322.

9. McAlister A, Vélez LF, De los Ríos R, Protocolo del Estudio Multicentrico Proyecto Activa. Investigación en Salud Publica, Documentos técnicos Organización Panamericana de la Salud, Washington, D.C; 1999. pp. 14-18.

10. Founier M, De los Ríos R, Orpinas P. Estudio Multicéntrico sobre Actitudes y Normas Culturales frente a la Violencia (Proyecto ACTIVA): Metodología. Rev Panam Salud Pública. 1999; 5(4/5): 222-231.

11. EPIINFO 6.04d. Centers for Disease Control and Prevention. Atlanta; 1996.

12. Statistical Packet for Social Sciences-SPSS. Paquete estadístico, $9^{\circ}$ versión. SPSS Inc.

13. Daniel W. Análisis de Varianza y Covarianza en Bioestadística base para el análisis de ciencias de la salud. Tercera edición. México DF: Editorial Limusa; 1997. pp. 345-451.

14. Hernández R, Fernández C, Baptista P. Metodología de la Investigación, Segunda edición, México: Mc Graw Hill;1998. pp. 255-266.

15. Ministerio de Salud, Centro de Investigación en Salud y Violencia de la Universidad del Valle: Cisalva, Modelo de Consentimiento informado para encuesta: Evaluación de creencias, actitudes y prácticas sobre violencia en CaliColombia;2 000.

16. Armitage P, Berry G. Análisis Avanzado de datos de regresión lineal en Estadística para la Investigación Biomédica. Versión en español de la tercera edición. Madrid: Harcourt Brace; 1997. pp. 285-294.

17. República de Colombia, Ministerio de Salud. Homicidios en Colombia en 1999. Semana epidemiológica No. 18. Informe Ejecutivo Semanal, Grupo Vigilancia en Salud Pública; 2000. pp. 1-6.

18. Vanegas G. Cali tras el rostro oculto de las violencias. Centro de Investigación en Salud y Violencia de la Universidad del Valle: Cisalva. Cali: Editorial de la Universidad del Valle;1998. p. 132.

19. Urrea F. Espacios regionales, movilidad y urbanización, dinámicas culturales e identidad de las poblaciones Afro colombianas del pacífico Sur y Cali. Una perspectiva integrada. Centro de Investigación Socioeconómica de la Universidad del Valle: CIDSE. Cali: Editorial de la Universidad del Valle; 2000.

20. Urrea F, Ortiz, C. Patrones sociodemográficos, pobreza y mercado laboral en Cali: Documento para el Banco Mundial, Centro de Investigación Socioeconómica de la Universidad del Valle: CIDSE. Cali: Editorial de la Universidad del Valle; 2000. 
21. Organización Panamericana de la Salud. La aplicación de teorías y técnicas de las ciencias sociales a la promoción de la salud. Rev Panam Salud Publica. 1998; 4(2):1-6.

22. Guzmán A. Violencia Urbana: teorías y políticas de seguridad Ciudadana. En: Armar la Paz desarmar la Guerra. Centro de estudios de la realidad Colombiana. Bogotá; 2000. pp.161-201.

23. Sarmiento A. Violencia y Equidad. En: Armar la Paz desarmar la Guerra. Centro de estudios de la realidad Colombiana, Bogotá; 2000. pp. 227- 261.

24. Orpinas P. Who is Violent?: Factors associated with aggressive behaviours in Latin America and Spain. Rev Panam Salud Pública. 1999; 4(4/5): 232-244.

25. Franco, S. Violencia y Salud en Colombia. Rev Panam Salud Publica. 1997; 1(2):93103.

26. Rubio, M. El desbordamiento de la Violencia en Colombia. En: Guerrero R, Londoño J (Ed.). Asalto al desarrollo: La Violencia en América Latina. Washington, D.C: Banco Interamericano de Desarrollo. 2000. pp. 101-167.

27. Buvinic M, Morrison A, Shifter M. Políticas para contrarrestar la Violencia en América Latina y el Caribe: Un Marco de referencia para la acción. Washington, D.C.; Banco Interamericano de Desarrollo. 1999. pp. 19-24.

28. McAlister A. La Violencia Juvenil en las Américas: Estudios innovadores de investigación, diagnóstico y prevención. Washington, D.C.: Organización Panamericana de la Salud y Agencia Sueca de Cooperación Internacional para el Desarrollo y Fundación W.K. Kellogg. 2000. pp.1-72.

29. Organización Panamericana de la Salud, Manual de Comunicación social para programas de promoción de la salud de los adolescentes, Washington, DC; 2001.pp. 24-27.

30. Dos Santos I, Epidemiología del Cáncer. Principios y Métodos, Agencia Internacional de Investigaciones Sobre el Cáncer, IARC Spress, Lyon, Francia; 1999.

31. Lozano R, Hijar M, Zurita B, Análisis de los costos y magnitud de la violencia en México. México: Banco Interamericano de Desarrollo y Fundación mexicana para la salud; 1997. pp. 28-40.

32. Concha-Eastman A. La violencia es prevenible. Un reto para los municipios. Programa de enfermedades no transmisibles, división de control y prevención de enfermedades, Organización panamericana de la Salud; 2000. 